\section{(A) OPEN ACCESS}

\title{
High rates of reinfection and incidence of bacterial sexually transmitted infections in a cohort of female sex workers from two Indian cities: need for different STI control strategies?
}

\author{
Anjana Das, ${ }^{1}$ Anupam Khungar Pathni, ${ }^{2}$ Prakash Narayanan, ${ }^{1}$ Bitra George, ${ }^{3}$ \\ Guy Morineau, ${ }^{4}$ Tobi Saidel, ${ }^{2}$ Parimi Prabhakar, ${ }^{1}$ Gururaj Rao Deshpande, ${ }^{5}$ \\ Raman Gangakhedkar, ${ }^{5}$ Sanjay Mehendale, ${ }^{6}$ Arun Risbud ${ }^{5}$
}

${ }^{1}$ STI Capacity Raising. FHI 360, New Delhi, India Independent Consultant New Delhi, India ${ }^{3}$ India Country Office, FHI 360, New Delhi, India ${ }^{4}$ Asia Pacific Regional Office, FHI 360, Bangkok, Thailand ${ }^{5}$ Department of Microbiology, National AIDS Research Institute, Pune, Maharashtra, India

${ }^{6}$ National Institute of Epidemiology, Chennai, Tamil Nadu, India

\section{Correspondence to} Anjana Das, STI Capacity Raising, FHI 360, H-5 Ground Floor, Green Park Extension, New Delhi 110016, India: anjanadas@fhi360.org

Received 6 January 2012 Revised 12 October 2012 Accepted 28 October 2012 Published Online First 29 November 2012

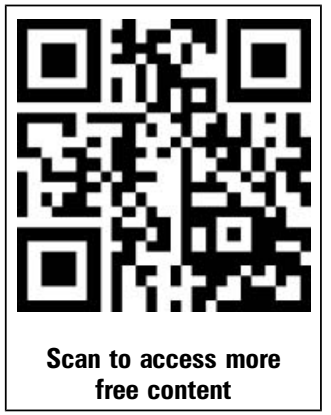

\section{ABSTRACT \\ Background Female sex workers (FSWs) in India are provided a standardised package of clinical interventions for management of sexually transmitted infections (STIs) A study was conducted among FSWs at known high STI prevalence sites to determine the effectiveness of the service package. \\ Methods A cohort of FSW clinic attendees in two cities, Hyderabad and Mumbai, were enrolled and followed up from October 2008 to November 2009. At each visit, behavioural and clinical data were obtained and vaginal swabs collected for laboratory testing of cervical infections (gonorrhoea and chlamydia).}

Results 417 participants were enrolled, of whom 360 attended at least a follow-up visit. Prevalence of cervical infections did not change between the baseline and final visits $(27.7 \%$ and $21.3 \%$ respectively, $p=0.08)$ in spite of presumptive treatment at baseline and syndromic management at all visits. The proportion of asymptomatic cervical infections increased from $36 \%$ at baseline to $77 \%$ at the final visit. Incidence rate of cervical infections was high (85.6/100 person years) and associated with a prevalent cervical infection at baseline $(\mathrm{HR}=2.7$, $p<0.001$ ) and inconsistent condom use with noncommercial partners ( $H R=2.5, p=0.014)$.

Conclusions High rates of STls persisted despite the interventions due to poor condom use, minimal partner treatment, and high prevalence and incidence of STIs with a large proportion of asymptomatic infections. Highprevalence FSW sites in India need to design more effective partner treatment strategies and consider increasing the frequency of presumptive treatment as a temporary measure for quickly reducing STI prevalence, with renewed emphasis on consistent condom use with all partners.

\section{BACKGROUND}

India is a large country with marked geographical heterogeneity in the prevalence of sexually transmitted infections (STIs), including HIV. ${ }^{1}{ }^{2}$ The HIV epidemic is mainly core group driven with a wide heterogeneity of HIV prevalence and stages of the epidemic across the country. ${ }^{3}{ }^{4}$ Predictably, the prevalence of classical STIs also varies across states and districts. ${ }^{1}$ However, the current state of knowledge is limited to primarily clinic-based studies, certain geographical areas and a wide variety of methods used for laboratory diagnosis. In the general population, various studies have shown that reactive syphilis serology ranged from 0 to $4.7 \%$ among women and from $1 \%$ to $10.1 \%$ among men. In female sex workers (FSWs), the range for reactive syphilis serology was $1.7-39.7 \%$, gonorrhoea $0-16.9 \%$, chlamydia $0.9-22.6 \%$ and trichomoniasis $2-54.1 \%{ }^{1}$

The Indian national STI control programme advocates syndromic management for the general population. ${ }^{5}$ In addition to syndromic management for symptomatic infections, FSWs are provided a package of services for managing asymptomatic infections, which includes one-time presumptive treatment for gonorrhoea and chlamydia at the first clinic visit, quarterly STI check-ups and biannual syphilis screening. In addition, risk reduction counselling, free provision of condoms, client-initiated partner notification and motivation for periodic HIV testing are integral parts of the standardised package of services for FSWs. ${ }^{6}$ However, these strategies were developed based on limited data from India and international best practices.

Avahan is a large-scale HIV prevention programme for high-risk groups (HRGs) which has been implemented since 2004 in 83 out of a total of 130 districts in six states of India with a high prevalence of HIV. Avahan works either alongside government or other donor-supported NGOs, or as the sole HIV prevention service provider for these groups in a district. Under this programme, approximately $221000 \mathrm{FSWs}$ are currently provided the STI service package as per national guidelines in conjunction with peer outreach to promote service uptake. ${ }^{7}$ To date, two rounds of community-based, district-wise surveys, the Integrated Biological and Behavioural Assessments (IBBA), have been carried out to evaluate the programme. The first round of the IBBA (IBBA-1) conducted in 2005-2007 showed an overall low prevalence of cervical infections in most districts; however, a higher prevalence was seen at FSW sites in Hyderabad (gonorrhoea 6.4\%, chlamydia 6.5\%) and Mumbai brothels (gonorrhoea $9.3 \%$, chlamydia $8.5 \%)^{8}$ Since STI interventions for FSWs at all districts had been initiated about a year prior to IBBA-1, the disparity in STI prevalence 
needed further investigation. An Avahan-supported operations research study was carried out among a cohort of FSWs in these two cities to determine the effectiveness of the standardised STI service package in controlling common bacterial STIs at known high prevalence sites. This paper analyses the findings and suggests additional STI management strategies for FSWs.

\section{METHODS}

The study design is depicted in figure 1. After written/oral witnessed consent at the baseline visit, trained female investigators administered a behavioural questionnaire covering demographics, sexual practices, condom use, symptoms and STI treatment seeking. Clinicians trained in study procedures carried out detailed history-taking and clinical examination of the anogenital area. Vaginal swabs were collected during speculum examination. Study participants were asked to return for follow-ups at 1, 2, 3 and 6 months after their initial visit. At follow-up visits during months 1,2 and 3 , a shortened version of the behavioural questionnaire pertaining only to risk behaviour was administered, clinical examination was performed and vaginal swabs were collected. The behavioural questionnaire and laboratory testing done at the final visit were the same as at the baseline visit. The study interventions included the Indian national STI service package for FSWs (mentioned earlier) and treatment for gonorrhoea and chlamydia for all at the baseline visit, either for the relevant syndrome (vaginal discharge (VD) or lower abdominal pain (LAP)) or presumptively. Presumptive treatment consisted of a single dose of cefixime $400 \mathrm{mg}$ and azithromycin $1 \mathrm{~g}$. Syndromic management was provided at all visits. Those with VD syndrome were treated for gonorrhoea (cefixime $400 \mathrm{mg}$ ), chlamydia (azithromycin $1 \mathrm{~g}$ ), candidiasis (fluconazole $150 \mathrm{mg}$ ), trichomoniasis and bacterial vaginosis (metronidazole $2 \mathrm{~g}$ ); those with LAP syndrome were treated for gonorrhoea (cefixime $400 \mathrm{mg}$ ), chlamydia (doxycycline $100 \mathrm{mg}$ twice daily for 14 days) and anaerobic infections (metronidazole $400 \mathrm{mg}$ twice daily for 14 days). Cefixime and azithromycin were administered under direct observation at the clinic. The treatment for other STI syndromes was as per the Avahan Clinic Operation Guidelines and Standards. ${ }^{9}$

Two study clinics were located in different parts of Hyderabad, providing services mainly to street and home-based sex workers. A third study site was located in a large brothel at Mumbai at a dedicated clinic for FSWs. The funding support for the outreach and clinical services varied across sites-while one of the Hyderabad sites was fully supported by Avahan, the other site was supported by Avahan for the clinical services and the National AIDS Control Program (NACP) for the outreach activities. The Mumbai site was supported by the NACP for the outreach services and the clinical services were provided through a local government clinic. From October 2008 to May 2009, all eligible and consenting attendees at these FSW clinics were recruited. Data collection of follow-up visits continued up to November 2009. The eligibility criteria for enrolment included at least two clients in the previous week and/or eight in the last month and age between 18 and 40 years. Additionally, pregnant women or those under the influence of alcohol/drugs at the time of consent were excluded from the study. Peer educators who were oriented about the study raised awareness during routine field visits and encouraged eligible FSWs to attend the clinic. Participants were given a card with their unique study number at enrolment on which the date of the next follow-up was written and explained to them at every visit. If participants provided a phone number, they were reminded about their appointments by a phone call from the study staff.

\section{Laboratory investigations}

Infections with Neisseria gonorrhoeae (GC) and Chlamydia trachomatis (CT) were detected in vaginal swab samples using transcription mediated amplification (Gen-Probe APTIMA Combo-2 Assay, Gen-Probe Inc, San Diego, CA, USA). Vaginal swabs were tested by the nucleic acid amplification technique using the PCR method published by Van Der Pol et a ${ }^{10}$ for Trichomonas vaginalis (TV). Sera from all participants were screened for syphilis by rapid plasma reagin (RPR, Span Diagnostics, Surat, India) and confirmation of all RPR-reactive sera was done by Treponema pallidum haemagglutination assay (TPHA) using Syphagen-TPHA (Biokit, Barcelona, Spain). An RPR titre of $\geq 1: 8$ with a positive TPHA was considered as an active syphilis infection.

\section{Ethical approval}

The study was approved by the Ethics Committee of the National AIDS Research Institute, Pune, India and the Protection of Human Subjects Committee of Family Health International, North Carolina, USA.

\section{Statistical analysis}

The questionnaire and clinical data of participants were entered into CSPro 3.3 (US Bureau of Census, USA). Participants with complete behavioural and biological data at baseline were included in the analysis. Analysis was performed using STATA V.12.0. The characteristics of participants lost to follow-up were compared with those retained in the cohort. Differences
Figure 1 Study design: visit schedule, study procedures and interventions among the female sex worker cohort. CT, Chlamydia trachomatis; GC, Neisseria gonorrhoeae; PCR, polymerase chain reaction; RPR, rapid plasma regain; TPHA, Treponema pallidum haemagglutination; TV, Trichomonas vaginalis. ${ }^{*} \mathrm{GCECT}$ treatment: cefixime $400 \mathrm{mg}+$ azithromicin $1 \mathrm{~g}$.

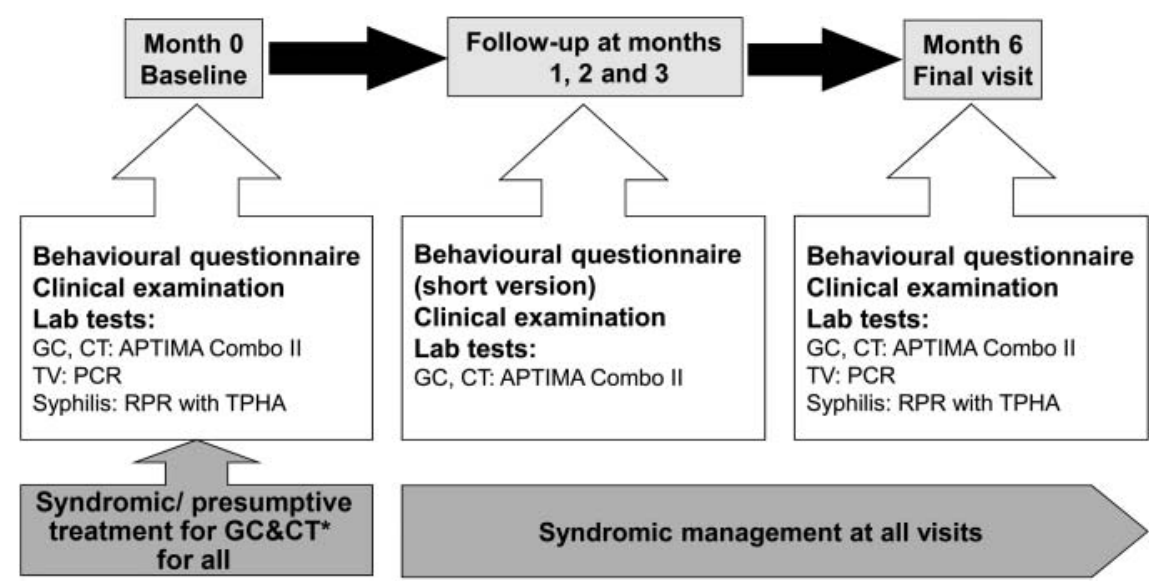


in behavioural characteristics and STI prevalence were assessed using the Wald test or the Fisher-exact test when cell size was less than 5 . The means of continuous variables were compared using the Student $t$ test. All tests were double sided and $p$ values $\leq 0.05$ were considered significant. Prevalence of GC, CT, TV and syphilis were compared at baseline and final visits among participants who attended the final visit (as per month 6 of figure 1). Asymptomatic infections were defined as laboratory-confirmed infections among individuals who did not complain of VD (TV, GC, CT) and/or LAP (GC, CT).

The principal outcome was the incidence of gonorrhoea and chlamydia. Lost to follow-up were those who were enrolled at baseline but did not attend any follow-up visits; they were excluded from the incidence analysis. Participants who had attended at least one follow-up visit with data on laboratory testing of GC and CT were included for the calculation of incidence of cervical infections. Incident cases were defined as GC and/or CT infections (by laboratory test) at the current visit among participants either uninfected or treated at the previous visit. Person years (PYs) for incidence rate (IR) calculations were measured by the time since the previous visit, assuming that the infection occurred at the mid-point between the visits. In addition, individuals who had received a single dose of azithromycin $1 \mathrm{~g}$ were considered to be protected against GC/CT for 7 days (because of the long half life of the drug) which was deducted from the person time.

Incidence of GC, CT, GC and/or CT was computed and comparison between those infected and those uninfected at baseline was assessed using Cox regression; hazard ratios (HRs) were presented. Predictors of GC/CT incidence were assessed using Cox regression. All variables associated with incidence of GC/CT in bivariate analysis with $p$ values $<0.2$ were included in a proportional hazard model. The final model was obtained using backward, stepwise elimination.

\section{RESULTS}

Of the 517 individuals screened during the recruitment period, 468 were eligible; 455 individuals consented to participate in the study, and of these, 417 completed all baseline study procedures. The demographic characteristics for those who refused to participate were not available. Among those who consented and completed $(n=417)$ and those who did not complete $(n=38)$ all baseline study procedures, there was no significant difference in socio-demographic characteristics except for the mean duration of sex work (4.6 and 6.3 years respectively, $p=0.047)$. Of the 417 individuals with complete data at baseline, 360 attended at least one follow-up visit. A total of 282 individuals attended the final visit (month 6 in figure 1).

The socio-demographic and behavioural characteristics, and STI prevalence were measured among all participants $(n=417)$ at baseline. Table 1 compares the baseline characteristics between those who attended at least one follow-up visit and those lost to follow-up. Those lost to follow-up were more likely to be brothel-based sex workers than those who attended follow-up $(23 \%$ vs $6 \%, p<0.001)$. Among all enrolled participants, the mean age was 30.4 years, the majority were streetbased sex workers (71\%), consistent condom use was low with both clients and non-commercial partners (70\% and $17 \%$ respectively), and TV was the most common STI (31\%). Further details about the baseline characteristics are discussed elsewhere. $^{11}$

At baseline, of a total of 417 participants, 270 (64.7\%) presented with complaints of VD and/or LAP, 234 (56.1\%) were treated for VD syndrome, while $59(14.1 \%)$ were treated for LAP syndrome. During 1089 follow-up visits, 198 episodes of VD and 44 episodes of LAP syndromes were treated.

Among 255 participants who made a follow-up visit within 90 days of baseline (when all were treated for GC and CT), the prevalence of GC and/or CT decreased from $25.5 \%$ to $14.4 \%$ $(p=0.002)$. For the 282 participants who attended the final visit, the mean number of days between the baseline and final visits was 217 days (SD 60). Between these visits, there was a significant increase in consistent condom use with clients from $69.5 \%$ to $80.9 \%(p=0.002)$ and a significant reduction in the mean number of clients in the last week from 4.7 (SD 3.4) to 3.4 (SD 2.5, p <0.001). However, consistent condom use with non-commercial partners remained low $(18.6 \%$ and $14.2 \%$ at baseline and final visits respectively, $p=0.214)$. The prevalence

Table 1 Characteristics of female sex workers at baseline and comparison of baseline characteristics between those lost to follow-up and those retained in the cohort

\begin{tabular}{|c|c|c|c|c|}
\hline Characteristics & $\begin{array}{l}\text { Attended at least one follow-up visit } \\
(n=360)\end{array}$ & $\begin{array}{l}\text { Lost to follow-up } \\
(n=57)\end{array}$ & p Value & $\begin{array}{l}\text { Total recruited at baseline } \\
(n=417)\end{array}$ \\
\hline Mean age in years (SD) & $30.4(4.7)$ & $30.0(6.6)$ & 0.531 & $30.4(5.0)$ \\
\hline \multicolumn{5}{|l|}{ Typology, \% (n) } \\
\hline Home & $22.5(81)$ & $10.5(6)$ & $<0.001$ & $20.9(87)$ \\
\hline Brothel & $5.8(21)$ & $22.8(13)$ & & $8.2(34)$ \\
\hline Street & $71.7(258)$ & $66.7(38)$ & & $71.0(296)$ \\
\hline Mean duration of sex work in years (SD) & $4.5(4.5)$ & $5.1(5.4)$ & 0.327 & $4.6(4.6)$ \\
\hline Mean number of clients past week (SD) & $4.8(3.5)$ & $5.2(3.7)$ & 0.392 & $4.9(3.5)$ \\
\hline Report consistent condom use with clients, \% (n) & $68.6(247)$ & $75.4(43)$ & 0.298 & $69.5(290)$ \\
\hline Currently have non-commercial partner/s, \% (n) & $69.7(251)$ & $73.7(42)$ & 0.543 & $70.3(293)$ \\
\hline Mean number of non-commercial partners (SD) & $1.3(0.7)$ & $1.2(0.7)$ & 0.542 & $1.3(0.7)$ \\
\hline Currently have more than one non-commercial partner, \% (n) & $22.8(57)$ & $22.5(9)$ & 0.966 & $22.8(66)$ \\
\hline Report consistent condom use with non-commercial partners, \% (n) & $17.5(44)$ & $14.3(6)$ & 0.605 & $17.1(50)$ \\
\hline \multicolumn{5}{|l|}{ Prevalence of STIs, \% (n) } \\
\hline $\mathrm{GC}$ & $14.4(52)$ & $12.3(7)$ & 0.663 & $14.2(59)$ \\
\hline CT & $15.0(54)$ & $22.8(13)$ & 0.136 & $16.1(67)$ \\
\hline $\mathrm{GC}$ and/or CT & $25.8(93)$ & $28.1(16)$ & 0.721 & $26.1(109)$ \\
\hline TV & $31.7(113)$ & $28.1(16)$ & 0.588 & $31.2(129)$ \\
\hline Active syphilis & $6.1(22)$ & $3.5(2)$ & 0.758 & $5.8(24)$ \\
\hline
\end{tabular}

CT, Chlamydia trachomatis; GC, Neisseria gonorrhoeae; STI, sexually transmitted infection; TV, Trichomonas vaginalis. 
Table 2 Prevalence of STls and proportion of infections without related symptoms among female sex workers who attended the final visit $(\mathrm{N}=282)$

\begin{tabular}{|c|c|c|c|c|c|c|}
\hline \multirow[b]{2}{*}{ Infection } & \multicolumn{3}{|c|}{ Prevalence } & \multicolumn{3}{|c|}{$\%$ Asymptomatic } \\
\hline & $\begin{array}{l}\text { Baseline } \\
\% \text { (n) }\end{array}$ & $\begin{array}{l}\text { Final visit } \\
\% \text { (n) }\end{array}$ & p Value & $\begin{array}{l}\text { Baseline } \\
\% \text { (n) }\end{array}$ & $\begin{array}{l}\text { Final visit } \\
\%(\mathbf{n})\end{array}$ & p Value \\
\hline GC & $15.3(43)$ & $14.9(42)$ & 0.906 & 34.9 (15) & $81.0(34)$ & $<0.001$ \\
\hline CT & $16.7(47)$ & $13.1(37)$ & 0.237 & $38.3(18)$ & $78.4(29)$ & $<0.001$ \\
\hline GC and/or CT & 27.7 (78) & $21.3(60)$ & 0.078 & $35.9(28)$ & $76.7(46)$ & $<0.001$ \\
\hline TV & $30.3(85)$ & 45.7 (129) & $<0.001$ & $29.4(25)$ & $77.5(100)$ & $<0.001$ \\
\hline Active syphilis & $6.8(19)$ & $2.5(7)$ & 0.016 & & & \\
\hline
\end{tabular}

CT, Chlamydia trachomatis; GC, Neisseria gonorrhoeae; STI, sexually transmitted infection; TV, Trichomonas vaginalis.

of STIs based on laboratory investigations at the baseline and final visits is shown in table 2 . Between the first and last visit, the prevalence of GC and/or CT reduced insignificantly from $27.7 \%$ to $21.3 \%$ (OR 0.71, $\mathrm{p}=0.078$ ), while TV increased from $30.3 \%$ to $45.7 \%$ (OR 1.94, $\mathrm{p}<0.001$ ). The proportion of asymptomatic infections was higher at the final visit than at baseline for GC, CT and TV (all p values <0.001). Seven incident cases of syphilis were observed at the final visit.

Of a total of 1089 follow-up visits, the intervals between consecutive visits were as follows: the majority (81\%) were within 90 days, $9.8 \%$ were within 180 days, while $8.5 \%$ were after 180 days. A total of 137 incident GC and/or CT infections were observed among 360 participants followed up for a period of 160.0 PYs. Accordingly, the IR for GC and/or CT infections was 85.6/100 PYs; calculated separately for GC and CT, it was 51.7/100 PYs and 56.8/100 PYs respectively. Higher IRs were observed among participants who had a cervical infection at baseline than those who were uninfected (table 3).

In multivariate analysis, the risk factors associated with incidence of GC and/or CTwere presence of infection at baseline (HR 2.8, $p<0.001)$, having a larger number of clients in the past week $(\mathrm{HR}=1.1$ per additional client, $\mathrm{p}=0.012)$, inconsistent condom use with non-commercial partners $(\mathrm{HR}=2.5, \mathrm{p}=0.014)$, follow-up visit to the clinic within 45 days compared with $>90$ days $(\mathrm{HR}=3.5, \mathrm{p}<0.001)$, and follow-up visit to clinic within 46-90 days compared with $>90$ days $(\mathrm{HR}=2.3, \mathrm{p}=0.002)$.

\section{DISCUSSION}

The study was conducted with a cohort of primarily streetbased FSWs who were provided standardised STI interventions. Among those who made a follow-up visit within 90 days of the baseline (when all were treated for GC and CT), the prevalence of GC and/or CT decreased from $25.5 \%$ to $14.4 \%$ $(p=0.002)$. However, owing to high IRs of GC and/or CT (85.6/100 PYs), there was no significant change in prevalence at baseline and final visits $(27.7 \%$ and $21.3 \%$ respectively, $p=0.08$ ). Higher IRs were associated with a prevalent cervical infection at baseline, inconsistent condom use with noncommercial partners and shorter duration between follow-up visits. The prevalence of TV increased between the baseline and final visits (from $30.1 \%$ to $45.7 \%, \mathrm{p}<0.001$ ) The proportion of asymptomatic GC, CT and TV infections increased to $80 \%$ at the final visit compared with $30-35 \%$ at baseline $(p<0.001)$.

To the best of our knowledge, evaluation of the effectiveness of a package of STI services among a cohort of FSWs in India has not been reported in the literature. However, the study had several limitations. It was conducted with a single group, which is not an optimal design for evaluating an intervention. More reliable conclusions would have been obtained from a randomised control trial. The study was conducted at sites with known high prevalence of GC/CT based on results of IBBA-1 (2005-2007). Hence the study findings may not be generalisable. As this was a clinic-based survey, the study group is likely to be biased towards symptomatic clients as against the general population of FSW. Collection of rectal and oral swabs for STI tests was not feasible, though $18.7 \%$ and $14.6 \%$ of participants at baseline did report oral and anal sex respectively in the preceding 3 months. Molecular tests for TV were performed only at the baseline and final visits; the incidence pattern of this common STI could have been determined if the tests were done at all follow-up visits.

High rates of STIs persisted despite the standardised intervention package (one-time presumptive treatment at baseline, clinical screening and syndromic management at all visits, risk reduction counselling, free condom provision and client-initiated partner notification). This was possibly due to poor rates of condom use, particularly with non-commercial partners; high baseline prevalence and incidence of cervical infections and trichomoniasis; and a high proportion of asymptomatic infections at follow-up visits. Although we did not track partner treatment in the study, inadequate partner treatment may have been a contributory factor to the high re-infection rates.

We compared our clinic-based findings on condom use with non-commercial partners and STI prevalence with other FSW studies from India. The IBBA-2 (2009-2010), performed after our study, also showed overall low rates of consistent condom use with regular partners ranging from $0.7 \%$ to $42 \%$ across districts. The prevalence of GC and CT in Hyderabad was $11.5 \%$ and $8.2 \%$, and among Mumbai brothel-based FSWs $5.9 \%$ and $4.1 \%$ respectively. ${ }^{12}$ The IBBA results show that some pockets of high prevalence persist in large cities like Hyderabad and Mumbai. Low condom use with regular, non-commercial partners has also been reported from other studies in India. ${ }^{13} 14$ A high prevalence of TV has been reported by several FSW studies in India. ${ }^{15} 16$ The current WHO global estimates show that TV constitutes more than half of all curable STIs. ${ }^{17}$

Our study showed a high incidence of GC and/or CT infections at the study sites (85.6/100 PYs), with two to three times

Table 3 Comparison of incidence rates (IRs) among female sex workers with cervical infections and those uninfected at baseline $(\mathrm{N}=360)$

\begin{tabular}{|c|c|c|c|c|c|c|c|c|c|c|}
\hline \multirow[b]{2}{*}{ Infection } & \multicolumn{4}{|c|}{ Not infected at baseline } & \multicolumn{4}{|c|}{ Infected at baseline } & \multirow[b]{2}{*}{ HRt } & \multirow{2}{*}{$\begin{array}{l}\text { Overall } \\
\text { IR (95\% CI) }\end{array}$} \\
\hline & $\mathbf{n}$ & Incidents & PYs & IR (95\% CI) & $\mathbf{n}$ & Incidents & PYs & IR (95\% CI) & & \\
\hline GC & 308 & 57 & 151.1 & 37.7 (29.1 to 48.9$)$ & 52 & 32 & 21.0 & 152.6 (107.9 to 215.8$)$ & $4.06^{*}$ & 51.7 (42.0 to 63.7 ) \\
\hline CT & 306 & 68 & 145.3 & 46.8 (36.9 to 59.4$)$ & 54 & 28 & 23.6 & 118.6 (81.9 to 171.8$)$ & $2.60^{*}$ & 56.8 (46.5 to 69.4$)$ \\
\hline GC and/or CT & 267 & 75 & 122.3 & $61.3(48.9$ to 76.9$)$ & 93 & 62 & 37.8 & $164.2(128.0$ to 210.6$)$ & $2.73^{*}$ & 85.6 (72.4 to 101.2$)$ \\
\hline
\end{tabular}

${ }^{*} p<0.001$

tComparison between incidence rates of those infected and uninfected at baseline.

CT, Chlamydia trachomatis; GC, Neisseria gonorrhoeae; IR, incidence rate; PY, person year. 
higher IRs among those with a prevalent infection compared with those uninfected at baseline. FSW studies from Kenya, Guatemala and Melbourne have reported lower IRs, ranging from 11.30 to $12.7 / 100$ PYs for gonorrhoea and from 7.32 to 14.5/100 PYs for chlamydia. ${ }^{18-20}$ Two FSW studies from Madagascar and China have reported very high IRs for gonorrhoea (0.09/person/month in Madagascar) and chlamydia (0.05/person/month in Madagascar and 65.91/PYs in China). ${ }^{21} 22$ Our findings are also similar to a study from Madagascar which reported that FSWs with a prevalent chlamydial, gonococcal or trichomonal infection at baseline were two to four times more likely to become infected during follow-up compared with those without STIs at baseline. ${ }^{23}$

The study showed an increasing trend of asymptomatic GC, CT and TV infections between the baseline and final visits. As this was a clinic-based study, the study group was likely to be biased towards symptomatic FSWs attending the clinic for treatment and recruited at baseline. In fact, about $65 \%$ of all participants recruited at baseline had symptoms of VD and/or LAP. A recent study carried out in five countries including a cohort of FSWs in India also showed an increasing trend of incident asymptomatic chlamydial infections over a period of 2 years $(31.2 \%$ from baseline to 12 months and $66.7 \%$ at 12-24 months). ${ }^{24}$ Thus, STI syndromic management would miss most cervical infections at follow-up visits owing to lack of symptoms, and laboratory facilities are unavailable at most FSW clinics.

The current practice in India for partner management is client-initiated partner notification. ${ }^{6}$ Although we did not track partner treatment in the study, the low condom use and early, high re-infection rates indicate that treatment for regular partners needs to be improved.

To examine the effectiveness of cefixime for the treatment of gonorrhoea, we searched for recent studies from India. A study of antimicrobial susceptibility of GC from India from 2002 to 2006 using ceftriaxone did not report resistance, but about 3\% of the isolates did show reduced susceptibility. ${ }^{25}$ All cases were given either ceftriaxone or cefixime and there were no treatment failures observed among those with the less susceptible strains. However, other countries have reported treatment failures with oral third-generation cephalosporin, which is a matter of concern. ${ }^{26}$

\section{Implications}

Based on our study, we have the following recommendations for the national STI programme in India. In addition to continuing efforts to promote consistent condom use, there is a need for designing more effective partner treatment strategies. Further studies are required, including expedited partner therapy, which has been proven to be useful in managing partners of heterosexual men and women with GC, CT and, to some extent, TV infections. ${ }^{27}$ One-off presumptive treatment may be inadequate at places with continuing poor STI control such as the study sites and proven active transmission dynamics, as shown by the high incidence. An increased frequency of presumptive treatment should be considered as a temporary measure for quickly reducing STI prevalence. ${ }^{28-31}$ This could be tapered off once the thresholds of $<10 \%$ combined GC/CT prevalence and $>70 \%$ reported condom use (with clients) have been met, as suggested by the 2005 WHO consultation. ${ }^{32}$ Addition of presumptive treatment for trichomoniasis, implemented elsewhere, may be considered. ${ }^{33}$ The availability of rapid, point-of care tests for GC/CT/TV would be very useful for screening of asymptomatic infections among FSWs at their initial clinic visit. This would enable the programme to intensify interventions, including retesting for those at high risk with a prevalent infection.

\section{Key messages}

- The female sex worker study was conducted at known high sexually transmitted infection (STI) prevalence sites to evaluate the effectiveness of a clinical service package for controlling STIs.

- The results showed a high incidence of cervical infections associated with a prevalent infection at baseline and inconsistent condom use with non-commercial partners.

- More effective partner treatment strategies need to be designed.

- High prevalence sites should consider increasing the frequency of presumptive treatment as a temporary measure.

Contributors $A D$ and $A K P$ were primarily responsible for drafting and revising the manuscript. $A D, P N$ and PP were responsible for the field research, oversaw data collection and management. GD and PP were responsible for the laboratory tests. PN, GM and TS contributed to the data analysis. AD, PP, BG, TS, SM, RG and AR participated in developing the concept and design of the study, data interpretation and manuscript review.

Funding This work was supported by the Bill \& Melinda Gates Foundation, grant number OPP31280. The views expressed herein are those of the authors and do not necessarily reflect the official policy or position of the Bill \& Melinda Gates Foundation.

Disclaimer The views expressed herein are those of the authors and do not necessarily reflect the official policy or position of the Bill \& Melinda Gates Foundation.

\section{Competing interests None.}

Patient consent Obtained.

Provenance and peer review Commissioned; externally peer reviewed.

Open Access This is an Open Access article distributed in accordance with the Creative Commons Attribution Non Commercial (CC BY-NC 3.0) license, which permits others to distribute, remix, adapt, build upon this work non-commercially, and license their derivative works on different terms, provided the original work is properly cited and the use is non-commercial. See: http://creativecommons.org/ licenses/by-nc/3.0/

\section{REFERENCES}

1. National AIDS Control Organization. Report on Mid-Term Review of Sexually Transmitted Infection Services. New Delhi, India: Department of AIDS Control, Ministry of Health \& Family Welfare, Government of India. December 2009. http://nacoonline.org/upload/STI\%20RTI\%20services/STI\%20RTI\%20MONOGRAPH\% 20 NACP-III-.pdf (accessed 11 Nov 2011).

2. National AIDS Control Organization. HIV Declining in India: New Infections Reduced by 50\% from 2000-2009. Sustained Focus on Prevention Required. Press release, New Delhi, India: Department of AIDS Control, Ministry of Health \& Family Welfare, Government of India. December 2010. http://nacoonline.org/upload/ HomePage/NACO\%2OPress\%20Release\%20on\%20HIV\%20Estimates.pdf laccessed 26 Dec 2011).

3. Nagelkerke NJ, Jha P, de Vlas SJ, et al. Modelling HIV/AIDS epidemics in Botswana and India: impact of interventions to prevent transmission. Bull World Health Organ 2002;80:89-96.

4. Chandrasekaran P, Dallabetta G, Loo V, et al. Containing HIV/AIDS in India: the unfinished agenda. Lancet Infect Dis 2006;6:508-21.

5. National AIDS Control Organization. National Guidelines on Prevention, Management and Control of Reproductive Tract Infections including Sexually Transmitted Infections. New Delhi, India: Ministry of Health \& Family Welfare, Government of India. August 2007. http://www.nacoonline.org/upload/STI\%20RTI\% 20services/National_Guidelines_on_PMC_of_RTI_Including_STI\%201.pdf laccessed 26 Dec 2011).

6. National AIDS Control Organization. Operational Guidelines for Programme Managers and Service Providers for Strengthening STI/RTI Services. New Delhi, India: Ministry of Health and Family Welfare, Government of India. October 2007. 
http://www.nacoonline.org/upload/STI\%20RT|\%20services/Operational\%20Guidelines \%20STI\%20RTI.pdf (accessed 26 Dec 2011)

7. Bill \& Melinda Gates Foundation. Treat and Prevent: Avahan's Experience in Scaling Up STI Services to Groups at High Risk of HIV Infection in India. New Delhi, India: Bill \& Melinda Gates Foundation. 2010. http://www.gatesfoundation.org/ avahan/Documents/treat-and-prevent.pdf (accessed 26 Dec 2011).

8. Indian Council of Medical Research and Family Health International. India Integrated Behavioural and Biological Assessment, Round 1 (2005-2007). National Summary Report. New Delhi, India: Indian Council of Medical Research and Family Health International. December 2009. http://www.fhi360.org/NR/rdonlyres/ ewxhge5nvpq2i4mjuo7vg2vnlzizg6rge7npe46jwrr2fwpjqp2vdv6lazazuj2oe7 lundg7gxthaf/IndiaNSRIBBAfnl010710.pdf (accessed 11 Nov 2011).

9. Family Health International. Clinic Operational Guidelines and Standards: Comprehensive STI Services for Sex Workers in Avahan Supported Clinics in India. India: Family Health International, 2007. http://www.fhi.org/en/HIVAIDS/pub/ res IndiaCOGs.htm (accessed 2 Nov 2010).

10. Van Der Pol B, Kraft CS, Williams JA. Use of an adaptation of a commercially available PCR assay aimed at diagnosis of chlamydia and gonorrhoea to detect trichomonas vaginalis in urogenital specimens. J Clin Microbiol 2006;44:366-73.

11. Das A, Prabhakar $P$, Narayanan $P$, et al. Prevalence and assessment of clinical management of sexually transmitted infections among female sex workers in two cities of India. Infect Dis Obstet Gynaecol 2011;2011:1-8. doi:10.1155/2011/ 494769

12. Indian Council of Medical Research and FHI 360. Integrated Behavioural and Biological Assessment, Round 2 (2009-2010). National Summary Report. New Delhi, India: Indian Council of Medical Research and FHI 360. July 2011. http://www.fhi360. org/en/HIVAIDS/pub/survreports/res IndialBBA2011.htm (accessed 8 Nov 2012).

13. Dandona R, Dandona L, Gutierrez JP, et al. High risk of HIV in non-brothel based female sex workers in India. BMC Public Health 2005;5:87.

14. Medhi GK, Mahanta J, Paranjpe RS, et al. Factors associated with HIV among female sex workers in a high HIV prevalent state of India. AIDS Care 2012;24:369-76

15. Desai VK, Kosambiya JK, Thakor HG, et al. Prevalence of sexually transmitted infections and performance of STI syndromes against aetiological diagnosis in female sex workers of red light area in Surat, India. Sex Transm Infec 2003;79:111-15.

16. Reza-Paul S, Battie T, Syed HU, et al. Declines in risk behaviour and STI prevalence following a community led HIV prevention intervention among female sex workers in Mysore, India. AIDS 2008;22(Suppl 5):S91-100.

17. World Health Organization. Prevalence and Incidence of Selected Sexually Transmitted Infections: Methods and Results Used by WHO to Generate 2005 Estimates. Geneva: Department of Reproductive Health and Research, World Health Organization, 2011. http://www.who.int/reproductivehealth/publications/rtis/ 9789241502450/en/ (accessed 26 Dec 2011).

18. Kaul R, Kimani J, Nagelkerke NJ, et al. Monthly antibiotic chemoprophylaxis and incidence of sexually transmitted infections and HIV-1 infection in Kenyan sex workers. JAMA 2004;291:2555-62.

19. Lee DM, Binger A, Hocking J, et al. The incidence of sexually transmitted infections among frequently screened sex workers in a decriminalized and regulated system in Melbourne. Sex Transm Infect 2005;81:434-6.
20. Sabido M, Giardina F, Hernandez G, et al. The UALE project: decline in the incidence of HIV and sexually transmitted infections and increase in the use of condoms among sex workers in Guatemala. J Acquir Immune Defic Syndr 2009; $\mathbf{5 1}$ (Suppl 1):S35-41.

21. Behets FM, Van Damme K, Rasamindrakotroka A, et al. Socio-demographic and behavioural factors associated with high incidence of sexually transmitted infections in female sex workers in Madagascar following presumptive therapy. Sex Health 2005; 2:77-84.

22. Ma S, Dukers NHTM, van den Hoek A, et al. Decreasing STD incidence and increasing condom use among Chinese sex workers following a short term intervention: a prospective cohort study. Sex Transm Infect 2002; 78:110-14.

23. Turner AN, Feldblum PJ, Hoke TH. Baseline infection with a sexually transmitted disease is highly predictive of reinfection during follow-up in Malagasy sex workers. Sex Transm Dis 2010;37:559-62.

24. Detels R, Green A, Klausner JD, et al. The incidence and correlates of symptomatic and asymptomatic chlamydia trachomatis and Neisseria gonorrhoeae infections in selected populations in five countries. Sex Transm Dis 2011;38:503-9.

25. Bala M, Ray K, Gupta SM, et al. Changing trends of antimicrobia susceptibility patterns of Neisseria gonorrhoeae in India and the emergence of ceftriaxone less susceptible $N$ gonorrhoeae strains. J Antimicrob Chemother 2007;60:587-93.

26. WHO Western Pacific and South East Asian Gonococcal Antimicrobial Surveillance Programmes. Surveillance of antibiotic resistance in Neisseria gonorrhoeae in the WHO Western Pacific and South East Asian Regions, 2009. Commun Dis Intell 2011;35:2-7.

27. Centers for Disease Control and Prevention. Expedited Partner Therapy in the Management of Sexually Transmitted Diseases. Atlanta, GA: US Department of Health and Human Services, 2006. http://www.cdc.gov/std/treatment/ EPTFinalReport2006.pdf (accessed 11 Nov 2011).

28. Steen R, Chersich M, de Vlas SJ. Periodic presumptive treatment of curable sexually transmitted infections among sex workers: recent experience with implementation. Curr Opin Infect Dis 2012;25:100-6.

29. Ramesh BM, Beattie T, Shajy I, et al. Changes in risk behaviours and prevalence of STls following HIV preventive interventions among female sex workers in five districts in Karnataka state, South India. Sex Transm Infect 2010;86:i17-24.

30. Van Dam J, Holmes KK. STD prevention: effectively reaching the core and bridge population with a four component intervention. Sex Transm Dis 2000;27:9-11.

31. Bollen L, Anartati AS, Morineau G, et al. Addressing the high prevalence of gonorrhoea and chlamydia among female sex workers in Indonesia: results of an enhanced, comprehensive intervention. Sex Transm Infect 2010;86:61-5.

32. World Health Organization. Periodic Presumptive Treatment for Sexually Transmitted Infections: Experiences from the Field and Recommendations for Research. Geneva: World Health Organization, 2008. http://whqlibdoc.who.int/ publications/2008/9789241597050 eng.pdf (accessed 11 Nov 2011).

33. Behets F, Rasolofomanana JR, Van Damme K, et al. Evidence-based treatment guidelines for sexually transmitted infections developed with and for sex workers. Trop Med Int Health 2003;8:61-5. 